\title{
COMPARAISON DES APPAREILS DE PÊCHE A L'ÉLECTRICITÉ EPMC et HÉRON
}

\author{
P. LAMARQUE, I. ARRIGNON* et C. GOSSET \\ Centre de Recherches Hydrobiologiques, \\ INRA, B.P. 3, Saint-Pèe-sur-Nivelle, 64310 ASCAIN
}

\section{RESUME}

Les performances de deux appareils de pêche à l'électricité, l'ElectroPuliman Moyen Couplable (EPMC) et le Héron, sont comparées à la fois sous l'angle de leurs caractéristiques et sous celui des quantités de poissons (8 espèces) capturées par unité de temps, dans une rivière homcgène. L'avantage revient au Héron.

\section{ABSTRACT}

\section{COMPARISON BETWEEN TWO ELECTRIC FISHING DEVICES, THE EPMC AND THE HERON}

The performances of two electric fishing apparatuses (EPMC and Heron) are compared with regard to their characteristics and the quantities of fishes (8 species) caught per time unit, in an homogeneous stream. The Heron fishing gear comes off best.

\footnotetext{
* Consell Supérieur de la Pêche (CSP) : 3, rue Sainte-Marie, 60200 Compiègne.
} 
 \\ COMPARAISON DES APPAREILS DE PECHE A L'ELECTRICITE EPMC ET HERON}

Le choix d'un appareil de pêche n'est pas une opération simple car de nombreux facteurs doivent être pris en considération : robustesse, maniabilité, prix, perfomances en matière de conductivité et, le plus important, l'efficacité de la pêche.

En 1969 (CHMIELEWSKI et al., 1973), une confrontation internationale devant permettre un tel classement avait eu lieu en Pologne, mais on s'en était tenu à classer des appareils par ordre d'efficacité. Pour cela, on avait déversé dans une rivière un nombre connu de truites et d'anguilles marquées et l'efficacité avait été fournie, pour chaque appareil, par le pourcentage de recaptures. Toutefois, la méthode employée était lourde et nous en utiliserons une autre.

Nous essayerons présentement de comparer les deux appareils EPMC, couplés en série ou en parallèle, avec le "Héron * alimenté par son groupe électrogène Titan-Kombi.

\section{CARACTER'STIQUES DES APPAREILS}

\subsection{PRESENTATION}

EPMC (fig. 1)

L'EPMC (CUINAT, 1965) constitue un appareil de pêche, couplable en série ou en parallèle avec un autre appareil de même type. II peut être utilisé en "solo" dans des eaux de conductivité moyenne, ou en "duo " quand les conditions de conductivité l'imposent : en parallèle dans les eaux de forte conductivité, en série dans les eaux de faible conductivité.

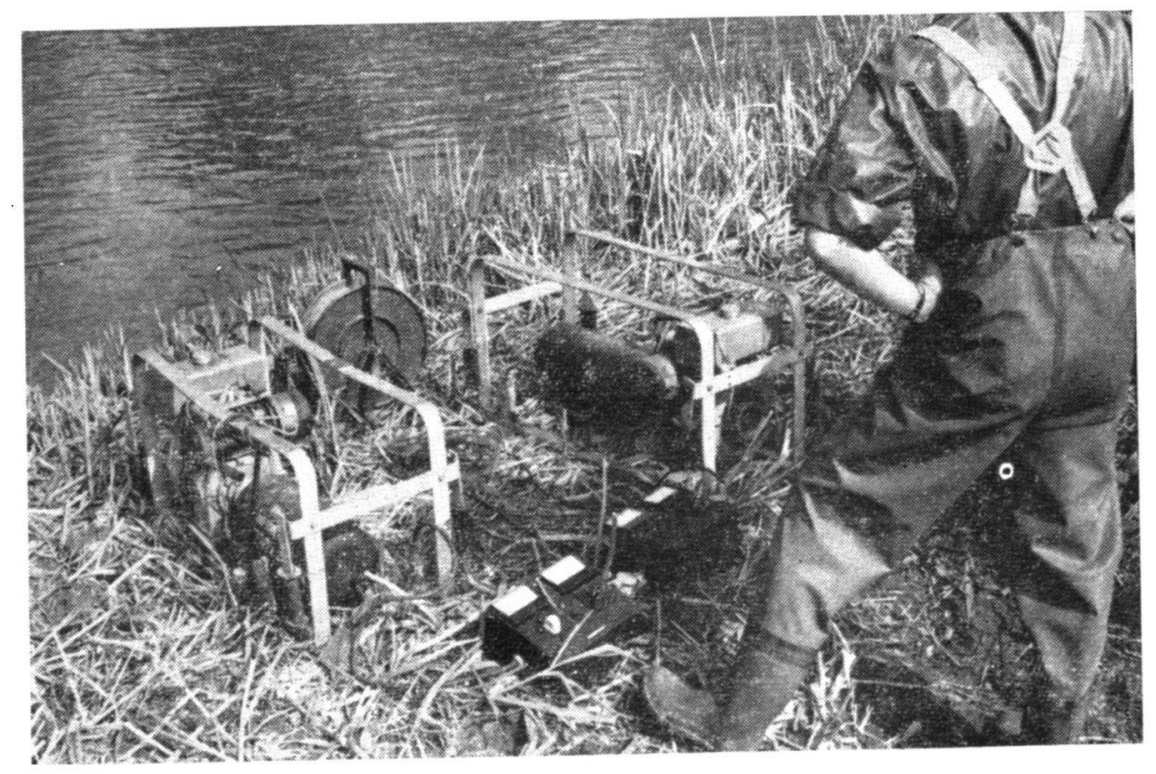

Figure 1 : L'appareil EPMC (Cliché Arrignon). 
Chaque appareil comprend un moteur (Bernard-Moteurs) et une dynamo (Electro-Pullman) fournissant du courant continu. Fonctionnant en a duo * ils sont reliés l'un à l'autre par une boite de raccordement qui comporte un rhéostat de réglage de l'excitation.

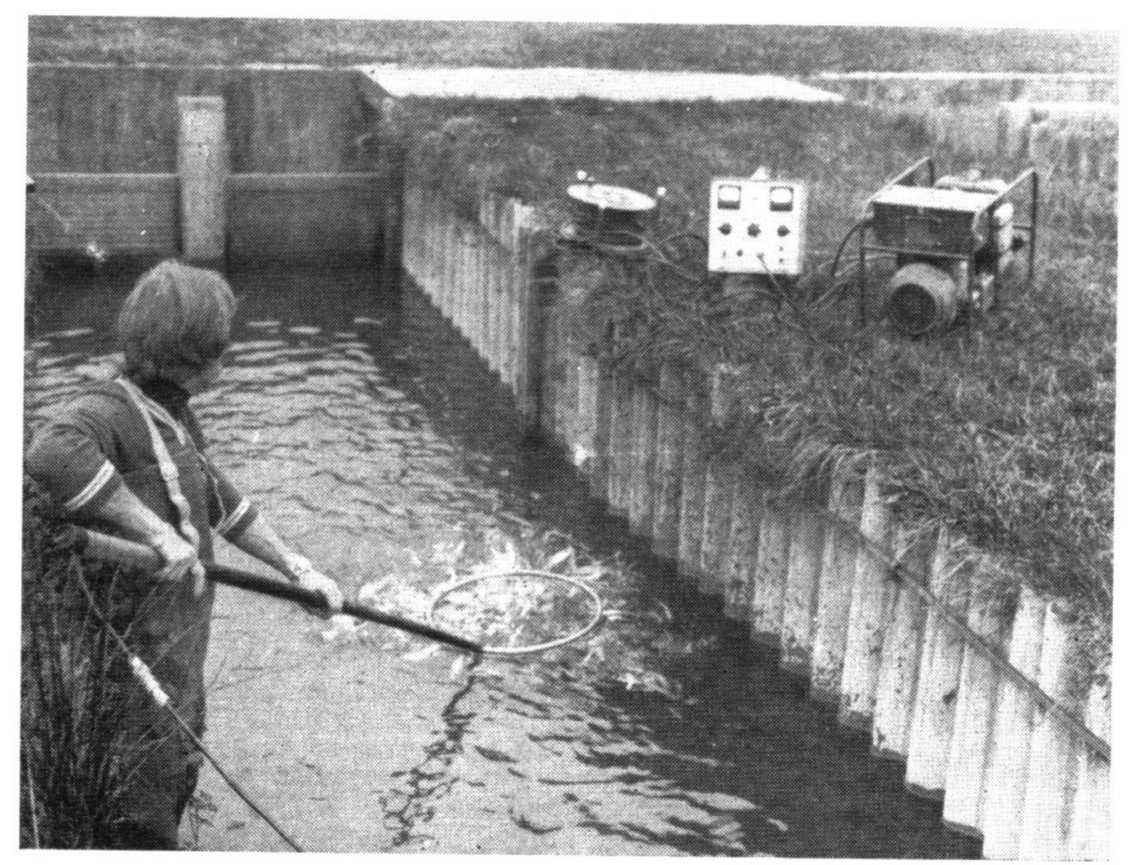

Figure 2 : L'appareil Héron (Cliché Claraz) : groupe électrogène Titan-Kombi et boítier de transformation.

HERON (fig. 2)

Le Héron (GOSSET, 1976) se présente sous l'aspect d'un boitier de commande comprenant principalement un autotransformateur à sorties multiples, une cellule de redressement et un circuit de commande à distance. Le générateur peut délivrer, à la demande :

- du courant continu sensiblement constant par redressement en pont (taux d'ondulation $4 \%$ ):

- du courant continu ondulé par redressement en étoile (taux d'ondulation $17 \%)$.

Le Héron a été conçu pour être alimentè par un groupe électrogène triphasé 220-380 V d'une puissance nominale de $3 \mathrm{kVa}$ à $\cos \phi=0,8$. En conséquence, dans l'étude qui suit, on entendra toujours par Héron l'ensemble groupe plus boitier de commande et les performances en puissance du boitier de commande en particulier seront étroitement liées aux possibilités du groupe choisi. Ce groupe Titan-Kombi que nous avons sélectionné possède l'inconvénient majeur d'être onéreux, mais en revanche il est d'une robustesse et d'une légèreté sans concurrence, donc parfaitement adapté aux conditions de terrain de la pêche à l'électricité. 
L'avantage du groupe électrogène sur la dynamo est également de pouvoir être utilisé, indépendamment de la pêche, pour l'éclairage, l'alimentation du petit outillage et les réparations sur le terrain (soudure).

II existe deux versions du Hèron (1 et 2) qui se différencient par la gamme de voltage (tabl. 1). Le Héron 2 doit remplacer le Héron 1 qui ne sera plus fabriqué.

\begin{tabular}{|c|c|}
\hline EPMC & Gammes de voltage (V) \\
\hline $\begin{array}{c}\text { Héron 1 } \\
\begin{array}{c}\text { courant presque continu } \\
\text { courant ondulé }\end{array}\end{array}$ & $\begin{array}{l}300,400,500,600 \text { et } 700(2630 \mathrm{~W} \text { crête }) \\
173,230,288,346 \text { et } 404(3520 \mathrm{~W} \text { crête })\end{array}$ \\
\hline $\begin{array}{c}\text { Héron 2 } \\
\text { courant presque continu } \\
\text { courant ondulé }\end{array}$ & $\begin{array}{c}170,210,260,330,410,510,640 \text { et } 800 \text { (2630 W crête) } \\
98,121,150,190,236,294,369 \text { et } 461 \text { (3520 W crête) }\end{array}$ \\
\hline
\end{tabular}

Tableau 1 : Performances en matière de voltage des appareils EPMC (2 appareils couplés) et Héron.

\subsection{POIDS ET ENCOMBREMENT}

La maniabilité en dépend. Le tableau 2 fournit ces caractéristiques.

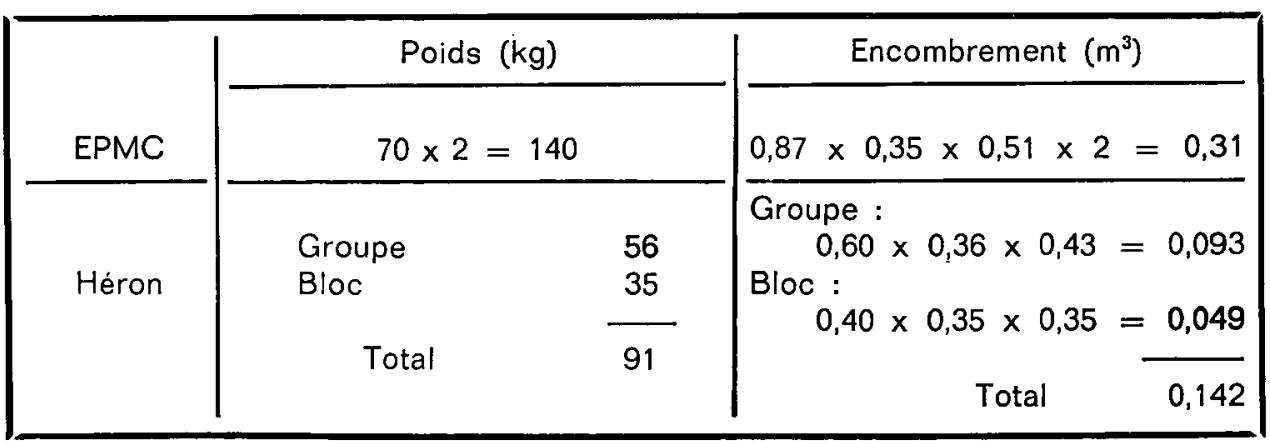

Tableau 2 : Poids et encombrement de l'EPMC (2 appareils couplès) et du Héron (groupe électrogène + bloc de transformation).

\subsection{RAPPORTS POIDS-PUISSANCE}

EPMC : 38,8

Héron : 32,5 (compte tenu du groupe électrogène)

\subsection{PRIX APPROXIMATIFS SANS ACCESSOIRES, HORS TAXES}

\section{EPMC}

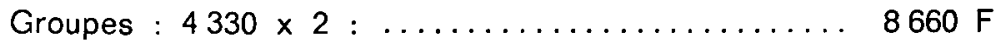

Tableau de commande $: \ldots \ldots \ldots \ldots \ldots \ldots \ldots . \ldots 2300 \mathrm{~F}$

Câbles de raccordement : ................ $230 \mathrm{~F}$

Total : $\quad 11190 \mathrm{~F}$ 
Héron

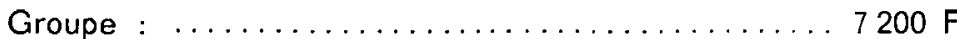

Boitier Héron : ................. $7800 \mathrm{~F}$ (Héron 2)

Total : $\quad 15000 \mathrm{~F}$

\subsection{TYPES DE COURANT}

\section{EPMC}

L'EPMC fournit du courant continu constant qui, jusqu'à présent, était considéré comme le meilleur courant pour la pêche.

Le Hèron fournit du courant sensiblement constant (presque continu) à taux d'ondulation de 4 p. 100 , et du courant ondulè à taux d'ondulation de 17 p. 100 .

Le courant sensiblement constant s'est révélé supérieur au courant continu : sans qu'il exerce une action tétanisante qui pourrait diminuer l'efficacité de la pêche, il permet d'abaisser le voltage nécessaire. Le voltage de crête minimal nécessaire à produire la nage vers l'anode (seuil), mesuré en laboratoire, esí en effet de 30 p. 100 inférieur à celui du courant continu (LAMARQUE, 1976). II en résulte que dans la pêche on peut également utiliser un moindre voltage pour produire le même effet.

Avec le courant ondule, le gain de voltage atteint 47 p. 100, mais ce courant exerce une action tétanisante qui peut diminuer l'efficacité de la pêche avec certaines espèces (anguilles), aussi peut-il être avantageux d'utiliser de préférence le courant sensiblement constant, lorsque c'est possible. En revanche, dans les eaux chaudes (To $>25$ ), le courant ondulé fournit de meilleurs résultats, pour des voltages inférieurs, que les courants continu ou sensiblement constant (GOSSET, 1976, donnèes non publiées).

\subsection{PERFORMANCES EN FONCTION DE LA CONDUCTIVITE DE L'EAU ET DE LA RESISTANCE INTER-ELECTRODES}

\subsection{Voltage}

Dans les eaux conductrices il faut utiliser un voltage relativement faible, superieur toutefois à $200 \mathrm{~V}$ avec le courant continu. Inversement, dans les eaux résistantes, il faut utiliser un voltage élevé qui peut atteindre ou dépasser $1000 \mathrm{~V}$ dans les eaux exceptionnellement résistantes. Ces voltages peuvent être abaissés avec le courant sensiblement constant et avec le courant ondulé.

Le tableau 1 fournit la gamme de voltage dont dispose chaque appareil (d'après CUINAT, 1965, pour les EPMC) et la figure 3 sa possibilité d'emploi en fonction de la résistance inter-électrodes.

On remarquera sur la figure 3 qu'au-dessus de 25 ohms environ de résistance inter-électrodes les voltages du Héron (courant sensiblement constant) sont légèrement inférieurs à ceux du courant continu fourni par les EPMC. Cette différence nous parait devoir être compensée par l'action plus efficace du courant sensiblement constant ( $\left.\begin{array}{l}\S \\ 1.5\end{array}\right)$. 

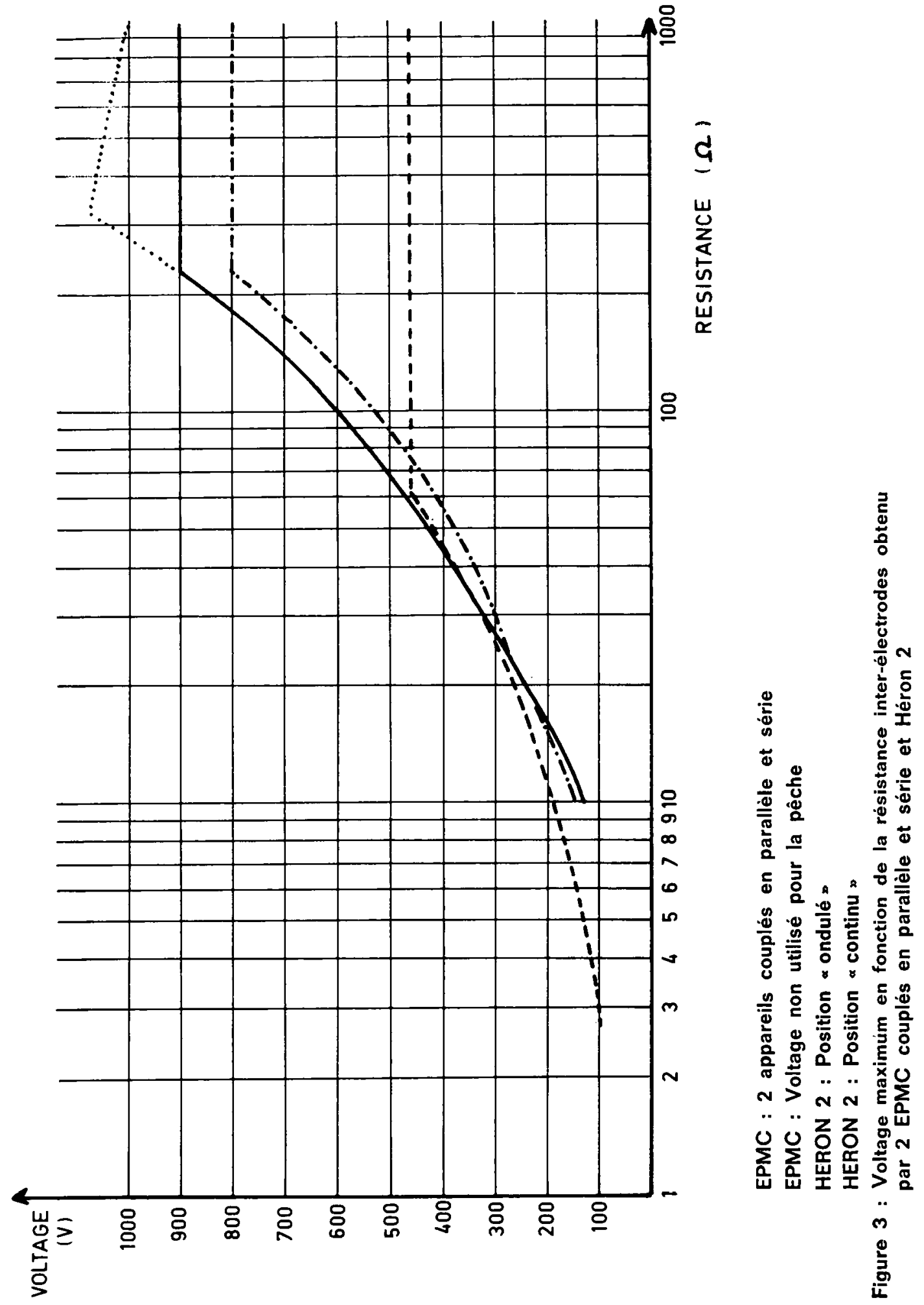
Par ailleurs, la tension du Héron a été limitée à $800 \mathrm{~V}$ mais rien ne s'oppose à ce que pour des cas d'eaux exceptionnellement résistantes, elle soit portée sur demande à une valeur supérieure. On notera sur la figure 3 qu'au-dessous de 25 ohms environ de résistance inter-électrodes, les voltages du courant sensiblement constant du Héron fournissent des tensions légèrement supérieures à celles des EPMC et surtout permettent de pêcher avec des valeurs de résistance inter-électrodes $(R \min =2,8$ ohms) beaucoup plus basses, la limite de fonctionnement correct des EPMC se situant à $10 \mathrm{ohms}$.

L'utilisation des faibles tensions sur les basses valeurs de résistance, sans trop nuire à l'efficacité de la pêche, n'est évidemment possible que grâce à l'action physiologique plus forte du courant ondulé dont les seuils d'action en voltage sont plus bas que ceux du courant continu (§ 1.5).

Nous ferons remarquer enfin que le réglage par palier de la tension de sortie du Héron aurait dû entrainer un diagramme de tension (fig. 3) également en palier. Cependant, les essais ont montré que le fonctionnement du groupe électrogène est satisfaisant même lorsque la résistance extérieure devient inférieure à la résistance normale minimale. Ce mode de fonctionnement dans une limite raisonnable entraine une chute de tension de sortie du groupe, mais pas de perte de puissance (fig. 4). Cela permet d'obtenir approximativement une variation continue de la tension d'utilisation.

\subsection{Puissance}

\section{EPMC}

CUINAT (1965, fig. 4) donne les courbes de puissance pour deux EPMC couplés en parallèle ou en série. On peut voir que la puissance maximale disponible varie beaucoup en fonction de la résistance extérieure. Dans le cas d'un couplage parallèle, la puissance nominale $(1800 \mathrm{~W} \times 2)$ n'est en fait disponible qu'à l'intérieur d'une plage étroite de résistances soit 50 à $100 \mathrm{ohms}$, alors que dans le cas d'un couplage en série cette plage se situe de 100 à 350 ohms environ. En dehors de ces limites cette puissance disponible décroit très rapidement.

CUINAT, par exemple, donne comme bonnes conditions de travail, dans le cas d'un couplage parallèle, les limites de résistances $20 \mathrm{ohms}$ et $150 \mathrm{ohms}$, soit $240 \mathrm{~V}, 12 \mathrm{~A}$ et $450 \mathrm{~V}, 3 \mathrm{~A}$. On voit immédiatement que, si, dans le premier cas, la puissance fournie est de $2880 \mathrm{~W}$, elle n'est plus que de $1350 \mathrm{~W}$ dans le second cas. II faut cependant remarquer que, placé dans des conditions d'utilisation optimales, l'ensemble peut fournir une puissance de 4,5 kilowatts.

\section{Héron}

Le groupe électrogène Titan-Kombi choisi délivre une puissance de $3 \mathrm{kVA}$ à $\cos \phi=0,8$ (désignation normalisée). II faut savoir que la puissance ohmique débitée par un groupe électrogène est constante, quel que soit le $\cos \phi$ de l'installation, et fixée par la puissance du moteur. Dans ce cas on aura donc $P=3 \mathrm{kVA} \times 0,8=2400 \mathrm{~W}$ aux pertes près dans le boitier de commande, ce sera donc la puissance disponible sur la résistance extérieure. Cependant, il faut également tenir compte du fait qu'il s'agit ici de puissance efficace et que les seuils de réaction du poisson sont déterminés en fonction des valeurs crêtes 


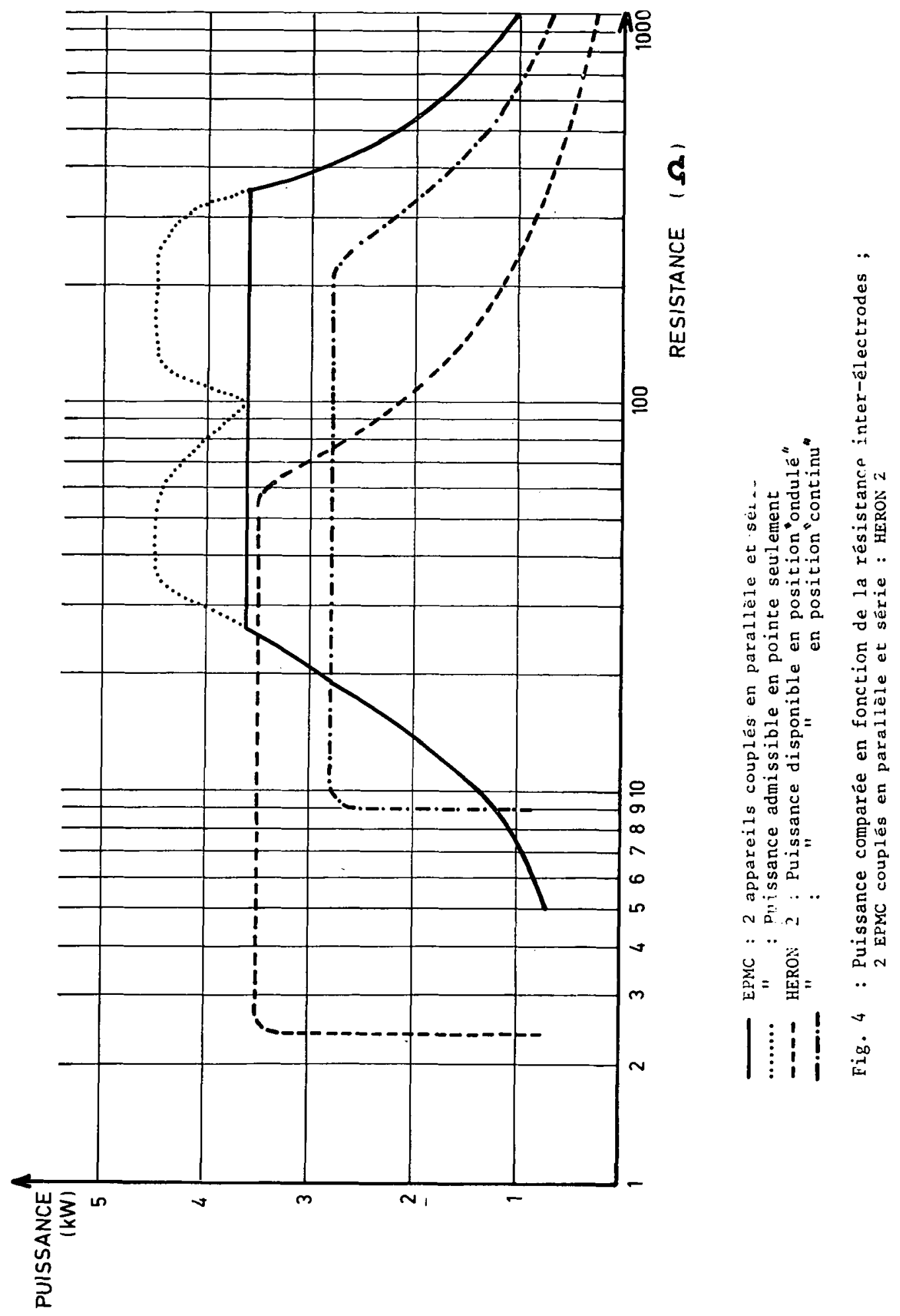


de la tension. C'est donc la puissance crête disponible qu'il faudra considerer. Prenons par exemple le cas du redressement en étoile où on a :

$$
\begin{aligned}
& V \text { eff. }=0,826 \mathrm{~V} \text { crête, dans ce cas on aura donc : } \\
& P \text { crête max. }(W)=\frac{P \text { eff. max. }(W)}{(0,826)^{2}}
\end{aligned}
$$

$$
\text { soit } P \text { crête max. }=\frac{2400}{(0,826)^{2}}=3520 \mathrm{~W} \text {. }
$$

Avec un redressement en pont cette différence est moins marquée puisque $\mathrm{V}$ eff. $=0,955 \mathrm{~V}$ crête mais, dans ce cas, le groupe fournit une puissance ohmique légèrement supérieure à sa puissance nominale : ceci peut être attribué à un débit plus régulier de l'alternateur.

La fig. 4 donne les puissances délivrèes par chaque appareil en fonction de la résistance inter-électrodes et du type de courant.

Au-dessus de 25 ohms la puissance délivrée par les EPMC est supérieure à celle du Héron. Mais nous ferons la même observation que nous avons faite pour le voltage : pour obtenir la même efficacité de pêche, le Héron nécessite une puissance moindre. Les écarts sont plus importants parce que la puissance dépend du carré du voltage.

Au-dessous de 25 ohms, la puissance délivrée par le Héron est supérieure.

Mentionnons enfin que le Héron, comme les EPMC, supporte des pointes de puissance au-dessus de la valeur nominale, ce qui est parfois nécessaire lorsque la conductivité du fond augmente ou lorsque l'électrode vient toucher une masse métallique.

\subsection{SECURITE}

\subsection{Protection des opérateurs}

\section{EPMC}

Avec cet appareil, l'anode est sous tension pendant toute la durée de la pêche. II en résulte qu'en cas d'incident près de l'électrode la coupure du courant ne peut ètre obtenue que par la déconnection de la prise au niveau de l'anode, ce qui prend un certain temps et constitue un danger du fait que cette prise est elle-même sous tension.

\section{Héron}

Dien qu'avec le Héron il soit également possible de pêcher sous tension constante, ce qui peut présenter certains avaritages, le manche de l'anode porte un contacteur de sécurité à pression douce sous faible tension ( $6 \mathrm{~V})$, qui commande un relais situé sur l'appareil. II suffit de relâcher la pression pour que le courant soit instantanément coupé.

Ce contacteur permet également de pratiquer un mode de pêche que nous recommandons particulièrement $\left(\begin{array}{ll}\S & 1.9\end{array}\right)$. 
II présente cependant deux inconvénients : d'une part, son étanchéité n'est pas parfaite bien que le fabricant le présente comme minirupteur étanche : il faut donc prendre des précautions pour qu'il ne prenne pas l'eau, ce qui pourrait avoir pour conséquence de le rendre conducteur; d'autre part, il nécessite l'emploi d'un cable à 3 conducteurs, plus lourd et par conséquent moins maniable qu'un monoconducteur.

Ces deux inconvénients peuvent être palliés par l'emploi d'un émetteur HF miniature commandant le courant. Dans cette éventualité, le contacteur est fixé sur le manche de l'anode et l'émetteur (500 g), ainsi que l'antenne, sur le dos du pêcheur. Le prototype a été réalisè et les premiers résultats sont satisfaisants. La portée actuelle est de $500 \mathrm{~m}$ et pourra atteindre $1000 \mathrm{~m}$ (prototype réalisé, mais non encore expérimenté sur le terrain). La majoration du prix de l'appareil ne devrait pas dépasser 600 francs.

Le courant du Héron est obtenu à partir d'un groupe électrogène de courant alternatif. Ce courant est plus dangereux que le courant continu. On peut donc se demander dans quelle mesure le danger pour les opérateurs ne s'en trouve pas augmenté.

D'une manière générale, qu'il s'agisse du Héron ou de l'EPMC, le danger près du groupe est limité si les précautions essentielles sont prises : port de cuissardes et signalisation. La question se pose surtout si le groupe est transporté sur un bateau d'où l'on pèche. En ce domaine, nous pouvons seulement faire état de notre expérience depuis 3 ans pendant lesquels le Héron a été utilisé dans des conditions parfois difficiles en Afrique, sur de petites embarcations (pirogues) où les opérateurs se tenaient dans sa proximité immédiate, sans que personne n'ait jamais reçu la moindre décharge. C'est sans doute dù à la très bonne qualité du groupe électrogène Titan-Kombi que nous recommandons, tant au point de vue robustesse qu'isolement.

\subsection{Protection de l'appareillage}

\section{EPMC}

En cas de court-circuit franc elle est assurée par un fusible. Mais il n'existe pas de protection contre les surcharges. CUINAT (1965) recommande de surveiller l'échauffement des dynamos. Or il est arrivé à notre connaissance que plusieurs dynamos ont été détruites par l'emploi de l'appareil dans une eau trop conductrice, les opérateurs n'ayant pas pris les précautions nécessaires.

\section{Héron}

En cas de court-circuit franc comme en cas de surcharge, le Hèron est protégé par un disjoncteur thermique.

\subsection{PANNES, ROBUSTESSE, ENTRETIEN, BRUIT}

Sur l'EPMC, les pannes résultent le plus souvent, si l'on excepte les mauvais emplois dont il a été fait état précédemment, des mauvais contacts aux charbons de la dynamo qu'il faut nettoyer ou changer.

Le succès de I'EPMC, avec quelque 250 appareils commercialisés depuis 1965, montre qu'il s'agit d'un appareil robuste. 
Les seules pannes observées depuis 3 ans sur le Héron ont résulté de mauvais branchements (innovations intempestives), de mauvais contacts aux prises (ce qui est également valable pour l'EPMC) et de mauvaise étancheité du contacteur de sécurité. Mais dans cette dernière hypothèse, il a toujours été possible de poursuivre la pêche en maintenant la tension constante.

Par ailleurs la conception du Héron est simple et son électronique relève de la compétence de n'importe quel électronicien. II peut donc être réparé localement.

A l'heure actuelle une dizaine de Héron sont en service et leurs utilisateurs en sont très satisfaits.

Le bruit des moteurs est également à prendre en considération, tant pour les opérateurs qui sont assourdis que pour les poissons qu'il peut faire fuir (gardons); or le bruit d'un seul EPMC est bien supérieur à celui du groupe électrogène du Hèron.

\subsection{MODES DE PECHE}

Nous terminerons le chapitre des caractéristiques des appareils en signalant que l'emploi d'un contacteur sur le manche de l'anode permet un mode de pêche qui nous parait plus efficace. En approchant l'anode des abris ou des poissons, on établit le courant au moment optimal. Ainsi on peut réduire les fuites qui se produisent à la limite du champ électrique actif. II permet aussi, par interruption du courant, de moins fatiguer les poissons qui se trouvent dans la proximité de l'électrode et qui n'ont pu être instantanément pêchés. L'opérateur peut également "manœuvrer" plus aisément les poissons pour les faire sortir de leurs abris (anguilles).

Le contacteur autorise enfin la capture à l'aide d'un filet revêtant l'anode, ce qui est parfois pratique, sans risque d'électrocuter les autres opérateurs en les touchant par mégarde.

\section{COMPARAISON DES EFFORTS DE PECHE}

Compte tenu des performances de voltage et de puissance du Héron, comparées à celle de l'EPMC, on aurait pu se passer de la présente comparaison.

Cependant, comme le courant du Héron est sensiblement différent de celui de l'EPMC, il était intéressant de l'effectuer.

\subsection{CONDITIONS DE LA COMPARAISON}

L'opération s'est déroulée avec le concours du Conseil Supérieur de la Pêche (Région Piscicole de Compiègne), dans le cadre d'un contrat de recherches CSP/INRA, dans un bras de la Risle (fig. 5).

Les caractéristiques de ce bras sont :

- largeur moyenne: $12 \mathrm{~m}$

- profondeur : de 0,5 à $1,5 \mathrm{~m}$

- conductivité : $476 \mu \mathrm{S} / \mathrm{cm}$ (2 100 ohms.cm)

- vitesse moyenne du courant d'eau et bonne visibilité. 


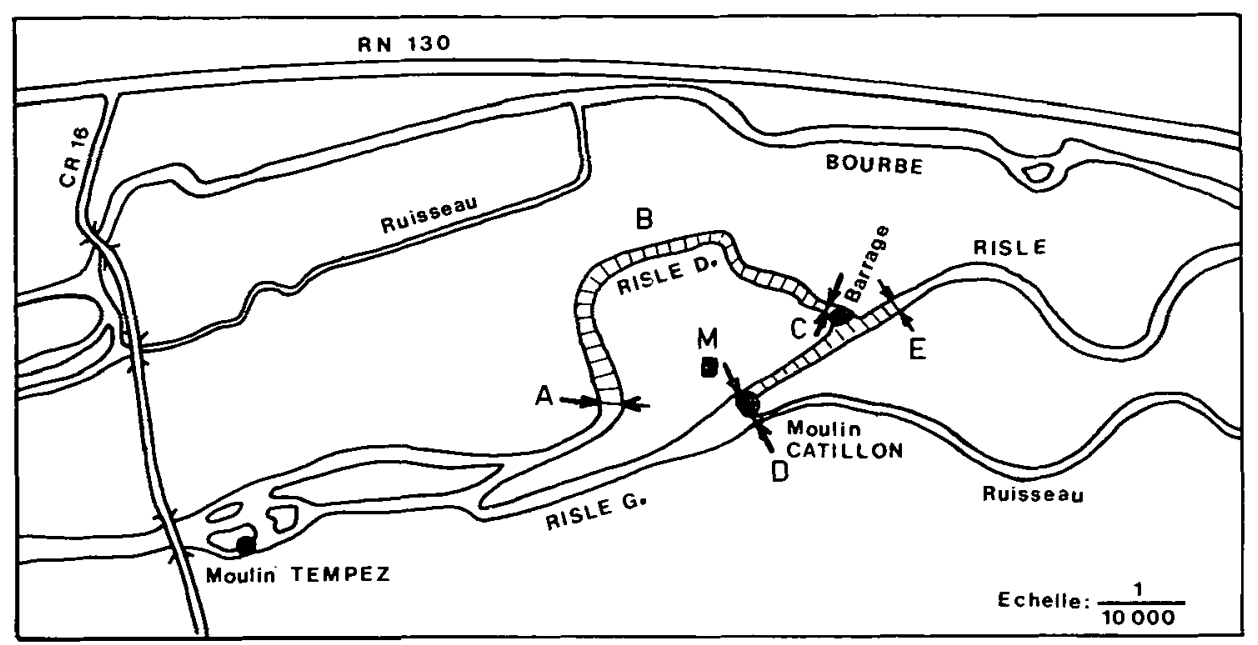

Fig. 5 : Emplacement de la comparaison des appareils

$$
\begin{aligned}
\mathrm{ABC} & : \text { ler parcours (secteurs } 1 \text { à } 11) \\
\mathrm{DE} . & \text { 2ème parcours (secteurs } 12 \text { à 16) } \\
\mathrm{M} & : \text { Emplacement des moteurs }
\end{aligned}
$$

Plutôt que de mesurer l'efficacité pour chaque appareil, ce qui eut allongé les opérations en nécessitant deux passages de pêche, il a été jugé préférable d'effectuer la comparaison en prenant en compte les quantités de poissons capturées toutes les dix minutes par chaque appareil.

L'équipe de pêche devait ignorer avec quel appareil elle pêchait, de manière que le comportement des pêcheurs ne puisse pas être influencé. A cette fin, les appareils étaient situés à plusieurs centaines de mètres du lieu de péche, afin qu'ils ne soient pas identifiables par le bruit du moteur. Toutes les dix minutes, un ordre ètait envoyé par radio, à partir du lieu de pêche, à l'emplacement des moteurs, pour que le courant soit interrompu. Les poissons capturés étaient alors identifiés, comptés et mesurés. Dès que cette opération était terminée, un nouvel ordre radio était envoyé pour que l'on change d'appareil. Seul le manipulateur des moteurs connaissait l'appareil avec lequel on pêchait.

II avait été en outre demandé aux pêcheurs de tenter de différencier les appareils par l'observation du comportement des poissons autour de l'anode.

Le Héron (courant sensiblement constant) et les deux EPMC branchés en parallèle avaient été réglés à 300 volts.

Dans ces conditions, l'intensité débitée était de $8 \mathrm{~A}$, ce qui correspondait à une résistance inter-électrodes de 37 ohms.

Dans les deux cas enfin, on avait utilisé les électrodes de l'EPMC, car il n'était pas possible de lui adapter le contacteur du Héron. 


\subsection{RESULTATS}

Le tableau 3 fournit les résultats de la comparaison.

Tableau 3 : Résultats des comparaisons d'appareils de pêche à l'èlectricité effectuées le 16 juin 1977 dans un bras de la Risle.

\begin{tabular}{|c|c|c|c|c|c|c|c|c|c|c|c|c|c|c|c|c|c|c|c|c|}
\hline & & EPS & & & & & & & & & & & & & & & Hér & on & & \\
\hline Parcours & $\begin{array}{l}1 \\
! \\
! \\
! \\
1\end{array}$ & & I & & & & 2 & $\begin{array}{l}1 \\
\text { II } \\
! \\
18 \\
!\end{array}$ & $\begin{array}{l}\text { Moyenne } \\
\text { par } \\
\text { secteur }\end{array}$ & $\begin{array}{r}! ! \\
! ! \\
! ! \\
! ! \\
! ! \\
\end{array}$ & & 1 & 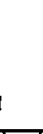 & & & 2 & & $\begin{array}{l}! \\
\text { !Moyenne } \\
! \quad \text { par } \\
\text { ! secteur } \\
!\end{array}$ & $\begin{array}{r}! \\
\text { ! } \\
! \\
\mathbf{r} \\
!\end{array}$ & $\begin{array}{l}\text { Cuservations et } \\
\text { niveau de signi- } \\
\text { ficativité des } \\
\text { différences }\end{array}$ \\
\hline $\mathrm{N}^{\circ}$ des secteur & $\begin{array}{l}! \\
5 ! \\
!\end{array}$ & 2 & 4 & 6 & 9. & IJ & 13 & $\begin{array}{r}15 ! \\
!\end{array}$ & & $\begin{array}{l}1 ! 3 \\
! !^{3} \\
! !\end{array}$ & s) & 7 & 8 & 10 & 12 & 14 & 16 & $\begin{array}{l}! \\
! \\
\end{array}$ & $!$ & \\
\hline $\begin{array}{l}\text { Nombre d } \\
\text { ANGUILLES } \\
\text { capturées } \\
20<L<75 \mathrm{~cm}\end{array}$ & $\begin{array}{l}! \\
! \\
! \\
!\end{array}$ & 10 & 15 & 18 & 19 & 6 & 41 & $\begin{array}{r}4 ! \\
\vdots \\
\vdots \\
\vdots\end{array}$ & 169 & $\begin{array}{l}1 ! \\
! 120 \\
1 ! \\
! ! \\
1 ! \\
\end{array}$ & $35 i$ & 21 & 32 & 26 & 27 & 49 & 38 & $\begin{array}{l}1 \\
! \\
\vdots \\
!\end{array}$ & $\begin{array}{l}! \\
! \\
! \\
!\end{array}$ & $\begin{array}{l}\text { Non.significatif } \\
t=1,56(\langle 2,16)\end{array}$ \\
\hline $\begin{array}{l}\text { Nombre de } \\
\text { BROCHETS } \\
\text { capturés } \\
25<\mathrm{L}<64 \mathrm{~cm} \\
\end{array}$ & $\begin{array}{ll} \\
! & 0 \\
! \\
! \\
1\end{array}$ & 1 & 2 & 0 & 1 & 0 & 2 & $\begin{array}{r}1 \\
1 \\
! \\
!\end{array}$ & 7 & $\begin{array}{ll}! \\
1 ! & 1 \\
1 ! \\
1 ! \\
1 !\end{array}$ & 0 & 0 & 0 & 1 & 0 & 0 & 0 & $\begin{array}{ll} \\
\vdots \\
!\end{array}$ & $\begin{array}{l}1 \\
\vdots \\
\vdots \\
\end{array}$ & N.S \\
\hline $\begin{array}{l}\text { Nombre de } \\
\text { ChEVAINES } \\
\text { capturés } \\
20<L<42 \mathrm{~cm} \\
\end{array}$ & $\begin{array}{l}1 \\
\vdots \\
\vdots \\
\vdots \\
1\end{array}$ & 2 & 1 & 1 & 5 & 13 & 1 & $\begin{array}{r}! \\
0 ! \\
! \\
!\end{array}$ & 23 & $\begin{array}{l}1 ! \\
1 ! \\
1 ! \\
! ! \\
! !\end{array}$ & 5 & 0 & 6 & 5 & 10 & 0 & 0 & $\begin{array}{ll}! & \\
! & 26 \\
! & \\
! & \\
\end{array}$ & $\begin{array}{l}1 \\
! \\
! \\
!\end{array}$ & N.S \\
\hline $\begin{array}{l}\text { Nombre de } \\
\text { GARDONS } \\
\text { capturés } \\
7<\mathrm{L}<34 \mathrm{~cm} \\
\end{array}$ & $\begin{array}{l}1 \\
! \\
\vdots \\
! \\
1\end{array}$ & 10 & 19 & 28 & $15 \mid$ & 21 & 5 & $\begin{array}{r}15 ! \\
! \\
! \\
\end{array}$ & $1 ! 9$ & $\begin{array}{l}! ! \\
! ! 28 \\
! ! \\
! ! \\
! !\end{array}$ & 6 & 62 & 13 & 25 & 11 & 16 & 8 & $\begin{array}{ll}! & \\
! & 169 \\
! & \\
! & \end{array}$ & $\begin{array}{l}! \\
! \\
! \\
!\end{array}$ & N.S \\
\hline $\begin{array}{l}\text { Nombre de } \\
\text { GouJONS. } \\
\text { capturés } \\
6<\mathrm{L}<21 \mathrm{~cm} \\
\end{array}$ & $\begin{array}{l}! \\
! \\
! \\
! \\
!\end{array}$ & 2 & 5 & 9 & 23 & 4 & 0 & $\begin{array}{r}! \\
0 ! \\
! \\
! \\
\end{array}$ & 13 & $\begin{array}{l}! \\
1 ! 3 \\
1 ! \\
! ! \\
1 !\end{array}$ & 0 & 13 & 3 & 7 & 5 & 0 & 2 & $\begin{array}{ll}! & \\
! & 33 \\
! & \\
& \\
\end{array}$ & $\begin{array}{l}! \\
! \\
! \\
!\end{array}$ & N.S \\
\hline $\begin{array}{l}\text { Nombre de } \\
\text { PERCHES } \\
\text { capturées } \\
14<\mathrm{L}<36 \mathrm{~cm} \\
\end{array}$ & $\begin{array}{l}7 \\
! \\
! \\
\vdots \\
!\end{array}$ & 3 & 0 & 2 & 3. & 3 & 0 & $\begin{array}{r}! \\
0 ! \\
! \\
!\end{array}$ & 13 & $\begin{array}{l}11 \\
110 \\
1 ! \\
1 ! \\
1 !\end{array}$ & 1) & 1 & 3 & 4 & 0 & 0 & 0 & $\begin{array}{l} \\
! \\
! \\
!\end{array}$ & $\begin{array}{l}5 \\
! \\
! \\
!\end{array}$ & N.S \\
\hline $\begin{array}{l}\text { Ombre de } \\
\text { TRUITES } \\
\text { capturées } \\
10<\mathrm{L}<51 \mathrm{~cm}\end{array}$ & $\begin{array}{l}1 \\
! \\
! \\
! \\
!\end{array}$ & 3 & 11 & 5 & 15 & 35 & 12 & $\begin{array}{r}! \\
16 ! \\
! \\
!\end{array}$ & 97 & $\begin{array}{ll}1 ! \\
1 ! 5 \\
1 ! \\
1 ! \\
! !\end{array}$ & 2 & 3 & 10 & 4 & 19 & 4 & 10 & $\begin{array}{ll}! \\
\vdots \\
\vdots \\
\vdots\end{array}$ & $\begin{array}{l} \\
! \\
! \\
!\end{array}$ & $\begin{array}{l}\text { N.S } \\
\text { Réserve pour les } \\
\text { truites du secteut } \\
2\end{array}$ \\
\hline $\begin{array}{l}\text { Nombre de } \\
\text { VANDOISES } \\
\text { capturées } \\
10<\mathrm{L}<20 \mathrm{~cm}\end{array}$ & $\begin{array}{l}7 \\
1 \\
1 \\
1 \\
!\end{array}$ & 2 & 0 & 0 & 9 & 7 & 0 & $\begin{array}{r}! \\
0 ! \\
! \\
\end{array}$ & 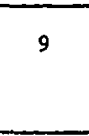 & $\begin{array}{ll}! \\
! ! & 0 \\
1 ! \\
! ! \\
! !\end{array}$ & 0 & 0 & 1 & 1 & 0 & 0 & 0 & $\begin{array}{ll} \\
! & 2 \\
! & \\
! & \end{array}$ & $\begin{array}{l}\vdots \\
\vdots \\
\vdots \\
1 \\
\end{array}$ & N.S \\
\hline $\begin{array}{l}\text { Nombre total } \\
\text { de porssons } \\
\text { capturés } \\
6<\mathrm{L}<75 \mathrm{~cm} \\
\end{array}$ & $\begin{array}{l} \\
! 24 \\
! \\
!\end{array}$ & 33 & 53 & 63. & 81 & 89 & 617 & $\begin{array}{r}76 ! \\
! \\
!\end{array}$ & 491 & $\begin{array}{l}11 \\
!: 57 \\
1 ! \\
! ! \\
! !\end{array}$ & isp & 00 & 68 & 73 & 72 & 69 & 58 & $\begin{array}{ll}! & \\
! & 546 \\
! & \\
\end{array}$ & $\begin{array}{l}1 \\
1 \\
1 \\
1\end{array}$ & $\begin{array}{c}N . S \\
t=0,66(<2,16)\end{array}$ \\
\hline
\end{tabular}


Huit secteurs, représentant chacun une pêche de $10 \mathrm{mn}$, ont été consacrés à chaque appareil. L'homogénéité des secteurs a paru bonne, à l'exception du secteur 2 où un seuil rocheux à l'aval d'un barrage constituait un biotope particulièrement favorable aux truites, mais relativement peu favorable aux anguilles, et du secteur 16, plus profond et plus difficile à pêcher.

Aucune différence significative entre les deux appareils n'apparait.

II semble toutefois qu'en matière de capture d'anguilles un léger avantage se manifeste au profit du Héron. En effet, sur l'ensemble des secteurs, la valeur de $t$ est de 1,56, ce qui indique que l'on a 85 chances sur 100 de ne pas se tromper en concluant que le Héron capture mieux les anguilles.

\section{CONCLUSIONS}

Si l'on récapitule les résultats de la comparaison, on peut retenir les èléments suivants.

\section{En matière de caractéristiques techniques :}

- en faveur de l'EPMC, le prix ainsi que la possibilité d'utiliser séparément les deux appareils lorsque la conductivité de l'eau le permet;

- en faveur du Héron, la maniabilité, la protection de l'appareillage, l'entretien, le bruit, la.sécurité des opérateurs (contacteur de sécurité), les possibilités d'amélioration (commande HF du courant), la possibilité de disposer d'un groupe électrogène pour d'autres usages que la pêche proprement dite.

\section{En matière de pêche :}

- pour une même * qualité pêchante» du courant sensiblement constant du Héron, cet appareil possède de plus grandes performances vers les plus fortes conductivités et vers le nombre d'anodes que l'on peut utiliser simultanément ;

- pour des "qualitès pêchantes" légèrement différentes — favorables dans certains cas, défavorables dans d'autres - ces performances sont encore accrues par la possibilité d'utiliser le courant ondulé du Héron.

Enfin, le Héron permet de pratiquer la pêche en commandant le courant, ce qui devrait en accroitre l'efficacité.

Tous les avantages que présente le Héron paraissent compenser largement la différence de prix.

Les EPMC ont permis pendant plus de 10 ans de pêcher de manière satisfaisante dans la majorité des eaux douces françaises : on peut donc regretter que la firme qui les produit n'ait pas su moderniser son appareillage. 


\section{BIBLIOGRAPHIE}

CHMIELEWSKI A., R. CUINAT, W. DEMBINSKI et P. LAMARQUE, 1973. Investigation of a method for comparing the efficiency of electric fishing machines. Pol. Arch. Hydrobiol., 20, 2, 319-340.

CUINAT R., 1965. L'appareil de pêche électrique Electro-Pullman Moyen Coupable (EMPC). Sta. Hydrobiol. contin. Biarritz, 15 pages ronéotypées.

GOSSET C., 1976. Un appareil de pêche à courant continu à haute performance : le Hèron. Piscic. fr., 47 : 25-29.

LAMARQUE P., 1976. Types de courant électrique à utiliser pour la capture optimale des poissons et principalement des anguilles. Piscic. fr., 47 : 30-37. 